\title{
Retrospective Study of the Seminalysis Report at an Infertility Clinic in South-Eastern Nigeria
}

\author{
Udokang Nsikak Ephraim, Udom Utibe Godwin
}

Department of Physiology, University of Uyo, Uyo, Nigeria

Email address:

nsikakudokang@yahoo.com (U. N. Ephraim)

To cite this article:

Udokang Nsikak Ephraim, Udom Utibe Godwin. Retrospective Study of the Seminalysis Report at an Infertility Clinic in South Eastern Nigeria. American Journal of Health Research. Vol. 7, No. 4, 2019, pp. 46-50. doi: 10.11648/j.ajhr.20190704.11

Received: June 26, 2019; Accepted: July 19, 2019; Published: August 6, 2019

\begin{abstract}
This study was carried out on 165 men in Nigeria. These men were husbands whose wives were attending infertility clinic in a hospital in Nigeria between the year 2013 and 2016. The semen parameters of these men were evaluated according to WHO (2010) procedures and they include semen volume, total sperm count, sperm concentration, sperm motility and semen $\mathrm{pH}$. The seminalysis recorded are as follows: Semen Volume (ml), 0-1.4 (20.6\%, 34men); 1.5-2.9 (34.0\%, 56men); 3.0 and above (45.4\%, 75men); Total Sperm Count (106/ml), 0-14.9 (18.8\%, 31men); 15-29.9 (27.2\%, 45men); 30 and above, $54.0 \%(89 \mathrm{men})$; Sperm Morphology (\%), 0-4, 3.7\%(6men); 5-50 (46.0\%, 76men); 51 and above (50.3\%, 83men); Progressive Sperm Motility (\%), 0-31 (44.2\%, 73men); 32-60 (29.7\%, 49men); 61 and above (26.1\%, 36men); Total Sperm Motility (\%), 0-39 (26.6\%, 44men), 40 and above $(73.4 \%, 121 \mathrm{men})$; Semen pH, less than 7.2 (49.1\%, 81men); $7.2-8$ (50.6\%, 84men). According to this result, and with direct reference to $49.1 \%$ of the subjects having abnormal semen $\mathrm{pH}$, it is concluded and in support of the claim that men contribute to almost half of the couples' infertility cases with abnormal sperm motility and semen $\mathrm{pH}$ being the highest contributing factors.
\end{abstract}

Keywords: Infertility, Male Infertility, Seminalysis

\section{Introduction}

In traditional Africa, the perception of marriage is solely about procreation and thus making infertility a marriage threat. Therefore, delay in conception in marriages brings about stigmatization as some people see infertility as a curse.

Apparently, men seem to presume that being able to achieve erection proves them fertile thus making the woman a prime suspect among infertile couples. In other to dispute this, women mostly give in for medical test but most men do not. This attests to the fact that infertile females face more social pressure in some countries like Nigeria than their male counterpart [1]. Though according to recent report, notable progress has been made in men coming to terms with the fact that infertility is a problem of couples [2].

According to the International Committee for Monitoring Assisted Reproductive Technology, World Health Organization (WHO) [3], infertility is a disease of reproductive system defined by failure to attain clinical pregnancy after 12 months or more of regular unprotected sexual intercourse. Infertility can be divided into primary infertility and secondary infertility. Infertility is primary if the couples are unable to get pregnant, while secondary infertility is the inability to get pregnant after an earlier pregnancy [4].

Infertility is a global health problem and in 2010 it was estimated that 48.5 million (45.0 million, 52.6 million) couples were infertile worldwide [5]. In WHO, (1999) [6], it had been shown that up to $15 \%$ of the population suffers with either primary or secondary infertility. In Nigeria and some parts of sub-Saharan Africa including the Republic of Sudan and Cameroon, infertility rate could exceed 30\% [7, 8]. Ikechebelu et al, (2003) [9], showed a $65 \%$ and $35 \%$ prevalent rate for primary and secondary infertility respectively. Sharlip et al., (2002) [10], reported that $50 \%$ of infertility cases are due to a solely female factor, pure male factor accounts for $20-30 \%$ of the problem, and the remaining $20-30 \%$ is due to a combination of both male and female factors.

According to Marimuthuet al., both males and females are equally responsible for the causes of infertility [11]. The 
major causes of infertility are identified to include testicular failure, obstruction, cryptorchidism, low semen volume, sperm agglutination, idiopathic infertility, varicocele, erectile or ejaculatory dysfunction, abnormal viscosity, endocrine disorder, high density of sperm, congenital abnormalities and environmental causes [12]. Sherins, observed that $50 \%$ cases of male infertility are idiopathic [13]. Sharma noted that factors that are implicated in male infertility can be divided into non-genetic and genetic factors and that among the nongenetic factors include previous exposure to disease which influences the fertility either directly or indirectly [14].

In this present study, the fertility profile of men whose partners were attending infertility clinic was evaluated using the following parameters: semen volume, total sperm count, sperm morphology, progressive motility, total motility and semen $\mathrm{pH}$.

The essence of this study is to compare these semen parameters to that of WHO (2010) [15] reference standard and toascertain the level of men's involvement in couples' infertility using this record.

\section{Methodology}

\subsection{Design}

This retrospective study was carried out on 165 men who were still within their reproductive age. These men of different professions were majorly Nigerians. They were men of couples attending infertility clinic within the period of 3 years (2013 to 2016) in a hospital in South- Eastern Nigeria.

Approval was gotten from the ethical committee of the hospital.

\subsection{Sample Collection}

The samples were collected in a private room near the laboratory, in order to limit the exposure of the semen to fluctuations in temperature and to control the time between collection and analysis.

Semen samples were collected by masturbation, after the men had been asked to abstain from ejaculation for at least 72 hours. The semen samples were collected into a clean graduated glass measuring cylinder with a wide mouth, from a batch that has been confirmed to be non-toxic for spermatozoa.

The specimen containers were kept at ambient temperature to avoid large changes in temperature that may affect the spermatozoa after they are ejaculated into it. The containers were numbered. The specimen containers were placed on the bench while the semen liquefies.

\subsection{Analysis}

The semen parameters of semen volume, total sperm count, sperm morphology, sperm motility and semen $\mathrm{pH}$ were evaluated.

\subsubsection{Semen Volume}

From the graduated glass measuring cylinder, the volume was read directly from the graduations ( $0.1 \mathrm{ml}$ accuracy).

\subsubsection{Total Sperm Count}

a) The semen and the prepared dilutions were mixed with fixative.

b) The haemocytometer chamber was loaded and the spermatozoa were allowed to settle in ahumid chamber.

c) The samples were assessed within 10-15 minutes.

d) Counting was done per replicate.

e) The replicate counts were compared to see if they are acceptably close before proceeding with the calculations.

f) The sperm concentration was calculated per ml. This was done by multiplying the volume of the total number of rows examined for the replicates by the dilution factor.

g) The total sperm count was obtained by multiplying the sperm concentration by the semen volume.

\subsubsection{Sperm Morphology}

a) A smear of semen was prepared on a slide.

b) The slide was air-dried, fixed and stained.

c) The slide was mounted with a coverslip.

d) Examination of the slide was done with bright field optics at $\times 1000$ magnification with oil Immersion

e) Assessment of approximately 200 spermatozoa per replicate was done for the percentage of normal and abnormal forms.

f) The replicate values were compared to see if they are acceptably close before proceeding with calculations.

\subsubsection{Progressive Sperm Motility}

a) The semen samples were mixed well.

b) An aliquot of semen was removed immediately after mixing, allowing no time for the spermatozoa to settle out of suspension.

c) The semen samples were remixed before removing a replicate aliquot.

d) For each replicate, a wet preparation approximately $20 \mu \mathrm{m}$ deep was prepared.

e) The samples were allowed to stop drifting before examination.

f) The slides were examined with phase-contrast optics at $\times 400$ magnification.

g) Approximately 200 spermatozoa were assessed per replicate for the percentage of different motile categories.

1) Progressive motility: spermatozoa moving actively, either linearly or in a large circle, regardless of speed.

2) Non-progressive motility: all other patterns of motility with an absence of progression, example, swimming in small circles, the flagellar force hardly displacing the head, or when only a flagellar beat can be observed.

3) Immotility (IM): no movement.

h) The replicate values were compared to check if they are acceptably close before proceeding with 
calculations.

\subsubsection{Total Sperm Motility}

Total sperm motility was calculated as Progressive motility + Non-progressive motility

\subsubsection{Semen pH}

a) $\mathrm{A} \mathrm{pH}$ paper in the range 6.0 to 10.0 was used.

b) The semen samples were mixed well.

c) A drop of semen was spread evenly onto the $\mathrm{pH}$ paper.

d) The colour of the impregnated zone was allowed to become uniform before reading.

e) The colour was compared with the calibration strip to read the $\mathrm{pH}$.

Details of these procedures have been published by WHO (2010) [15].

\section{Results}

Table 1. Semen analysis.

\begin{tabular}{|lll|l}
\hline Parameters & Ranges & $\begin{array}{l}\text { Number of } \\
\text { subjects }\end{array}$ & $\begin{array}{l}\text { Percentage of } \\
\text { subject }\end{array}$ \\
\hline Semen volume $(\mathrm{ml})$ & $0-1.4$ & 34 & 20.6 \\
& $1.5-2.9$ & 56 & 34.0 \\
& 3.0 -above & 75 & 45.4 \\
Total Sperm Count $\left(10^{6} / \mathrm{ml}\right)$ & $0-14.9$ & 31 & 18.8 \\
& $15-29.9$ & 45 & 27.2 \\
& 30 -above & 89 & 54.0 \\
Sperm Morphology (\%) & $0-4$ & 6 & 3.7 \\
& $5-50$ & 76 & 46.0 \\
Progressive Sperm Motility & $51-$ above & 83 & 50.3 \\
(\%) & $0-31$ & 73 & 44.2 \\
& $32-60$ & 49 & 29.7 \\
Total Sperm Motility (\%) & $0-39$ & 44 & 26.1 \\
& 40 -above & 121 & 26.6 \\
\hline Semen pH & Less than & 81 & 73.4 \\
& 7.2 & 49.1 \\
\hline
\end{tabular}

WHO (2010) [15] reference standard of semen volume $1.5 \mathrm{ml}$ and above, total sperm count of $15 \mathrm{million} / \mathrm{ml}$ and above, sperm morphology of $4 \%$ and above, progressive motility of $32 \%$ and above, total motility of $40 \%$ and above and semen $\mathrm{pH}$ of above 7.2 were considered normal.

The results showed that $79.4 \%$ of the subjects had semen volume within the normal range while that of $20.6 \%$ of the subjects were below the normal range. $81.2 \%$ of the subjects had total sperm count within the normal range while $18.8 \%$ of the subjects recorded below normal range. The sperm morphology results showed that $96.3 \%$ of the subjects were within the normal range while that of $3.7 \%$ being below normal range. Progressive sperm motility results revealed that $55.8 \%$ of the subjects were within the normal range while $44.2 \%$ were below the normal range. $73.4 \%$ of subjects had total sperm motility within the normal range while that of $26.6 \%$ of the subjects were below the normal range. Semen $\mathrm{pH}$ result revealed that $50.9 \%$ of the subjects were within the normal range while $49.1 \%$ had below normal range.

\section{Discussion}

Men's contribution to couples' infertility has over the years been questioned; if they do not or contribute less significantly to this health problem. This question arose due to the societal misconception in some countries that infertility was solely a woman's problem and the fact that more women visit specialists mostly and earlier on this problem than men.

Ekwere revealed in his research in Calabar, Nigeria that males take almost twice longer time to consult a specialist on this issue than their female counterparts [2]. This makes the literature on prevalence, causes, treatment and standard of reference of male infertility limited and unreliable in some cases.

With semen volume of $1.5 \mathrm{ml}$ per ejaculate as the lowest reference according to the WHO (2010), 79.4\% of the population is considered as being fertile. But can the rest of the population be actually termed infertile? This population could have either increased or decreased as some studies suggest lesser reference limit while others recommend higher limit. These controversies make the evaluation of male fertility using semen analysis seem unreliable and misleading with reference to any standard.

Spermatogenesis as a function of sex hormones and the testis itself contributes greatly to the total sperm in the semen, though its transport along the tract is of essence too. According to Chemes and Rawe, the etiology of male infertility in the Nigerian population study seems to be related to the influence of genital mycoplasma and ureaplasma species on the sperm count [16]. Infections in the male genito-urinary tract including infections of the epididymis, seminal vesicles, prostate, bladder and urethra are known to play a major role in many cases of infertility [17]. It could be considered therefore, that sex hormonal imbalance contributes greatly to the low sperm of spermatogenesis origin and infection lowers sperm count during the delivery phase. Considering this, it is likely that the sperm count value obtained when there is disruption in spermatogenesis phase may differ from that which is obtained when this disruption occur in the delivery phase.

Abnormal spermatozoa morphology has been linked not only to a decrease in traditional parameters of semen quality $[18,19]$ but has also been observed to be accompanied by reactive oxygen species (ROS) overproduction which further damages the spermatozoa, ultimately leading to infertility[20]. The spermatozoa with $<4 \%$ normal morphology were considered inviable in this study and $3.7 \%$ of the subjects had sperm morphology within this range. This may suggest that abnormal sperm morphology contributes less significantly to male infertility but its occurrence should not be neglected as its effect on fertilization could be more debilitating than other anomalies.

Said et al. wrote that a man with completely immotile sperm is sterile as immotile sperm whether living or dead cannot penetrate cervical mucus [21]. From the result of 
this study, $26.6 \%$ of the subject produced dead sperm cells or immotile sperm cells (from total motility evaluation) and $44.2 \%$ produced either slow or non-motile sperm cells (from progressive motility evaluation). With this high percentage of subjects with abnormal motility coupled with the fact that sperm motility has been seen as one of the most important parameter in evaluating male fertility potential, it is based on this that it could be presumed that abnormal sperm motility contributes more significantly to male infertility.

Singh et al. deduced that sperm $\mathrm{pH}$ affects sperm motility and capacitation. Infection has also been seen to be implicated in abnormal semen $\mathrm{pH}$. The correlation between semen $\mathrm{pH}$ and sperm motility is also observed in this work in a close range value of $44.2 \%$ for subjects producing slow or non-motile sperm and $49.1 \%$ having an abnormal semen $\mathrm{pH}$ value. This high percentage is actually placing abnormal semen $\mathrm{pH}$ contribution to male infertility on the high side.

Using WHO (2010) [15] reference standard, the result of this study shows that among the tested semen parameters, none recorded $100 \%$ fertility among the subjects. This proves men involvement in couples' infertility but the fact that this standard may be misleading should not be overruled. This is because this reference ranges and reference limits were provided using the semen quality of fertile men, whose partners had a time to pregnancy of 12 months or less from fathers in eight countries in three continents, and its use as globally standard may be unreliable.

\section{Conclusion}

Infertility test, using semen analysis with WHO standard reference could be unreliable, since the data used by WHO to arrive at these values did not cover the whole population; and geographical conditions contribute a lot to reproductive health. Therefore, providing reference standards according to geographical regions could be more efficient.

With the WHO (2010) standard reference, it could be postulated that men are not without blame in couples' infertility as observed in this study that none of the tested parameters has it being a $100 \%$ fertility record among them.

As revealed in this study, the abnormality of semen parameters in men's infertility in order of decreasing prevalence rate is as follows; semen $\mathrm{pH}$, sperm motility, sperm number, semen volume and finally sperm morphology. Infection could also be pointed out as one of the most common causes since it has been observed to be implicated in the parameter with high degree of contribution.

From this current work and with direct reference to $49.1 \%$ of the subjects having abnormal semen $\mathrm{pH}$, we therefore conclude and in support of the claim that men contribute to almost half of the couples' infertility cases.

\section{References}

[1] Ekwere PD, Archibong EI, Bassey EE, Ekabua JE, Ekanem EI, Feyi Waboso P (2007). Infertility among Nigerian Couplesas seen in Calabar, Port Harcourt Med. J. 2: 35-40.

[2] Ekwere, P. D (2004). Profile of sub-fertile Nigerian men in an infertility clinic in Calabar, Nigeria. Niger J Surg. 10: 3742.

[3] Zegers-Hochschild F, Adamson GD, De Mouzon J, Ishihara O, Mansour R, et al. (2009) International Committee for Monitoring Assisted Reproductive Technology (ICMART) and the World Health Organization (WHO) revised glossary of ART terminology, 2009. FertilSteril 92: 1520-1524.

[4] Panti AA, Sununu YT. The profile of infertility in a teaching Hospital in North West Nigeria. Sahel Med J 2014; 17: 7-11.

[5] Mascarenhas MN, Flaxman SR, Boerma T, Vanderpoel S, Stevens GA (2012) National, Regional, and Global Trends in Infertility Prevalence Since 1990: A Systematic Analysis of 277 Health Surveys. PLoS Med 9 (12): e1001356. doi:10.1371/journal. pmed.1001356.

[6] WHO (1999) Laboratory manual for the examination of human semen and sperm-cervical mucus interaction (4th edn), Geneva, World Health Organization, Switzerland.

[7] Larsen U (2000) Primary and secondary infertility in subSaharan Africa. Int J Epidemiol 29: 285-291.

[8] Okonofua EF, Harris D, Odebiyi A, Kane T, Snow RC (1997) Thesocial meaning of infertility in Southwest Nigeria. Health TransRev 7: 205-220.

[9] Ikechebelu JI, Adinma JI, Orie EF, Ikegwuonu SO (2003) High prevalence of male infertility in South-eastern Nigeria. J Obstet Gynaecol 23: 657-659.

[10] Sharlip I. D., Jarow J. P., Belker A. M., Lipshultz L. I., Sigman M., Thomas A. J., Schlegel P. N., Howads S. S., Nehra A., Damewood M. D., Overstreet J. W. and Sadovsky R. Best practice policies for male infertility. Fertil Steril. 2002; 77: 873-82.

[11] Marimuthu P, Kapilashrami MC, Misro MM, Singh G. Evaluation of trend in semen analysis for 11 years in subjects attending a fertility clinic in India. Asian J Androl. 2003; 5: 221-5. [PubMed].

[12] Makker K, AgarwalA, Sharma R (2009) Oxidativestress and male infertility. Indian J Med Res 129: 357-367.

[13] Sherins RJ (1995) How is male infertility defined? In: Handbook of Andrology, Robaire B, Pryor JL and Trasler JM (eds.) Lawrence: American Society of Andrology. Allen Press, USA. pp. 48-51.

[14] Sharma A (2017) Male Infertility; Evidences, Risk Factors, Causes, Diagnosis and Management in Human. Ann Clin Lab Res Vol. 5. No. 3: 188.

[15] World Health Organization Department of Reproductive Health and Research (2010) World Health Organization Laboratory Manual for the Examination and Processing of Human Semen, (5th edn). Geneva, Switzerland. 
[16] Chemes EH, Rawe YV: Sperm pathology: a step beyond descriptive morphology. Origin, characterization and fertility potential of abnormal sperm phenotypes in infertile men. Hum Reprod Update 2003, 9:405-428.

[17] Skowronek F, Casanova G, Alciaturi J, Capurro A, Cantu L, Montes JM, Sapiro R: DNA sperm damage correlates with nuclear ultrastructural sperm defects in teratozoospermic men. Andrologia 2012, 44: 59-65.

[18] Agarwal, A., Tvrda E. and Sharmaet, R. Relationship amongst teratozoospermia, seminal oxidative stressandmaleinfertility. Reprod Biol Endocrinol. 2014; 12: 45.
[19] Singh, A. K., Tomar, S., Chaudhari, A. R. and Singh, R. Sperm motility index and it's relation to sperm concentration in subjects with impaired fertility potential Biomedical Research 2010; 21 (1): 59-62.

[20] Tampion D, Gibbons RA: Swimming rate of bull spermatozoa invarious media and the effect of dilution. $J$ ReprodFertil1963; 5: 259-275

[21] Said TM, Agarwal A, Sharma RK, Mascha E, Sikka SC, Thomas AJ Jr: Human sperm superoxide anion generation and correlation with semen quality in patients with male infertility. Fertil Steril 2004, 82: 871-877. 